\title{
MICROCIRURGIA ENDOSCÓPICA TRANSANAL NO TRATAMENTO DOS TUMORES DO RETO: estudo prospectivo em 50 pacientes
}

\author{
Roberto da Silveira MORAES ${ }^{1}$, Osvaldo MALAFAIA ${ }^{1}$, José Ederaldo Queiroz TELLES², \\ Marcus Adriano TRIPPIA ${ }^{3}$, Gerhard F. BUESS ${ }^{4}$ e Júlio Cezar Uilli COELHO
}

\begin{abstract}
RESUMO - Racional - A literatura médica aceita ressecção local como opção válida em casos selecionados de tumores de reto. A seleção dos pacientes requer exata estimativa dos riscos e avaliação pré-operatória precisa, tanto no aspecto clínico como histopatológico. Objetivo Avaliar os resultados da microcirurgia endoscópica transanal em seguimento de 18 meses. Métodos - De abril de 2002 a abril de 2006 , 50 pacientes com tumores de reto foram submetidos a microcirurgia endoscópica transanal, selecionados por suas características clínicas e histopatológicas. Os critérios de inclusão foram: adenomas sésseis maiores do que $3 \mathrm{~cm}$ e menores do que $8 \mathrm{~cm}$ não-circunferenciais; neoplasia intra-epitelial de alto grau; carcinoma retal pT1, e em casos especiais pT2. Todos esses tumores foram submetidos ao mesmo procedimento cirúrgico. Resultados - O resultado histopatológico final revelou 9 adenomas, 26 neoplasias intra-epiteliais de alto grau, 13 carcinomas ( 9 pT1 e 4 pT2) e 2 carcinóides. A menor idade foi de 25 anos e a maior de 92 . O menor tumor ressecado tinha $64 \mathrm{~mm}^{2}$ (carcinóide) e o maior (adenoma) $90 \mathrm{~mm}^{2}$. O tempo médio de operação foi de 90 minutos e o de internamento 5 dias. Houve uma morte não relacionada ao método. Um paciente com carcinoma de baixo risco pT1 apresentou recidiva 18 meses após microcirurgia endoscópica transanal e foi submetido a retossigmoidectomia curativa. Foi observado tumor residual em dois pacientes e a complicação mais grave foi uma fístula retovaginal. A taxa de complicação global foi de $9 \%$. Conclusão - Atualmente a microcirurgia endoscópica transanal é a técnica de escolha para o tratamento de adenomas sésseis, neoplasias intra-epiteliais de alto grau e carcinoma retal pT1 de baixo risco.
\end{abstract}

DESCRITORES - Neoplasias retais, cirurgia. Microcirurgia. Proctoscopia.

\section{INTRODUÇÃO}

A polipectomia endoscópica e a excisão local para o adenoma e o carcinoma do reto tiveram recentemente aumento crescente na sua popularidade. Entretanto, é necessária seleção criteriosa dos doentes quando se deseja tratamento curativo.

Para se indicar o tratamento local de um carcinoma retal, sem a realização de linfadenectomia, deve-se cotejar o risco de metástases linfonodais existentes e também a mortalidade da ressecção radical, especialmente nos $\operatorname{idosos}^{(10)}$. Outros aspectos essenciais incluem avaliação histológica minuciosa, seguimento rigoroso e a advertência ao paciente da possível necessidade de ressecção radical curativa adicional.

$\mathrm{O}$ tratamento local do câncer retal não é recente. O primeiro acesso do reto para esse fim da qual se tem noticia foi praticada por Lisfranc em 1826. Entretanto, ele relatou elevada incidência de incontinência fecal, recidiva tumoral precoce e mortalidade alta, e referiu ser tudo isso relacionado à anatomia da pelve que dificultava a realização do tratamento local. Desde então surgiram várias modificações nas técnicas de ressecção transanal ${ }^{(25)}$.

Embora a ressecção dos pólipos pediculados com alças de polipectomia já esteja bem definida na literatura, as lesões sésseis maiores do que $4 \mathrm{~cm}$, o carcinoma pT1 e, em casos escolhidos, o pT2 permaneciam, até o advento da microcirurgia endoscópica transanal (TEM), necessitando de procedimentos mais invasivos ${ }^{(15)}$. Atualmente aceitase que a ressecção local possa ser indicada em casos selecionados de câncer retal ${ }^{(1,3,4,5,6,17,18,19,20,21,23,28,29)}$. Entretanto, há discordância sobre a facilidade e a qualidade da exposição do campo operatório com as varias técnicas descritas para esse fim. A dificuldade de boa exposição

Trabalho realizado no Serviço de Cirurgia do Aparelho Digestivo do Hospital de Clínicas da Universidade Federal do Paraná (UFPR), Curitiba, PR.

Serviços de ${ }^{1}$ Cirurgia do Aparelho Digestivo da UFPR; ${ }^{2}$ Anatomia Patológica da UFPR; ${ }^{3}$ Radiologia da UFPR; ${ }^{4}$ Department of General Surgery, Eberhard-Karls University, Tuebingen, Alemanha.

Correspondência: Dr. Roberto da Silveira Moraes - Rua Major Heitor Guimarães, 1925 -80740-340 - Curitiba, PR. E-mail: rdsmoraes@ig.com.br 
com os retratores convencionais, a exposição ruim dos tumores quando se afastam da linha pectínea, as altas taxas de complicações e morbidade geral das operações de Mason, Kraske e Parks, associadas aos inconvenientes quando da ressecção anterior e a mutilação da operação de $\operatorname{Miles}^{(7,14,17)}$, serviram de estímulo para o desenvolvimento de novo método cirúrgico, combinando a boa visualização proporcionada pela endoscopia, com o avanço tecnológico do instrumental cirúrgico para a ressecção minimamente invasiva.

Esse método foi denominado de microcirurgia endoscópica transanal (TEM) para diferenciá-lo do procedimento endoscópico e do cirúrgico convencional ${ }^{(2,3)}$. Passou a ser praticado a partir de 1982 na Alemanha, sendo realizado atualmente em grandes centros. Sua introdução no Brasil deveu-se ao primeiro autor deste trabalho em 2002 no Serviço de Cirurgia do Aparelho Digestivo no Hospital de Clinicas da Universidade Federal do Paraná (HCUFPR) em Curitiba, $\mathrm{PR}^{(21)}$.

\section{MÉTODOS}

De abril de 2002 a abril de 2006 foram operados pela mesma equipe 50 pacientes com tumores localizados no reto (Figura 1), com idades de 25 anos a 92 anos, em estudo prospectivo nãorandômico, aprovado pela Comissão de Ética do HCUFPR e registrado no B1NPESQ - UFPR (Banco de pesquisa da UFPR) sob número 2002012334.

\begin{tabular}{lc}
\hline Adenomas & 9 \\
Neoplasia intra-epitelial de alto grau & 26 \\
Carcinoma & 13 \\
pT1 & 9 \\
pT2 & 4 \\
Carcinóide & 2 \\
Recidiva & 1 \\
Residual & 2 \\
Ressecção anterior curativa & 1 \\
Mortalidade & 1 \\
Taxa de conversão & 0 \\
Total & 50 \\
\hline
\end{tabular}

FIGURA 1. Casuística de pacientes com tumores de reto submetidos a endoscopia transanal no período de abril de 2002 a abril de 2006

Os dados de entrevista foram registrados em protocolo padrão de atendimento que relacionava a história clínica, os antecedentes mórbidos pessoais e familiares e aqueles possivelmente relacionados à doença. Também foram pesquisados e registrados dados detalhados das características da lesão, dos exames e observações de interesse do pré, bem como os do trans e pós-operatórios. Foram candidatos a realizar a TEM os pacientes com tumores retais (adenomas sésseis maiores do que 3 e menores do que $8 \mathrm{~cm}$ não-circunferenciais, as neoplasias intra-epiteliais de alto grau, o carcinoma pT1 e, em casos escolhidos, os pT2).

Os doentes participantes do protocolo foram orientados, através de um termo de consentimento livre e esclarecido, sobre as características do procedimento a que seriam submetidos, das suas vantagens, desvantagens, benefícios e riscos.
No pré-operatório, todos os pacientes foram submetidos a toque retal com o objetivo, nos tocáveis, de verificar extensão mobilidade e localização na circunferência retal; retossigmoidoscopia rígida com objetivo de se realizar macrobiopsias e, fundamentalmente, de se determinar a distância do tumor da linha pectínea e da margem anal e a sua localização na parede do reto, se anterior, posterior, lateral esquerda ou lateral direita. Os limites de ressecção estabelecidos foram de $12 \mathrm{~cm}$ na parede anterior, $15 \mathrm{~cm}$ nas paredes laterais e $20 \mathrm{~cm}$ na parede posterior. A ressecção total da parede era realizada em todos os adenomas sésseis maiores do que $4 \mathrm{~cm}$ e naqueles com diagnóstico pré-operatório de carcinomas localizados no reto extraperitonial.

Também, realizava-se colonoscopia e/ou enema opaco para verificação de lesões associadas e doenças concomitantes, ultrasonografia endorretal, ultra-sonografia abdominal, avaliação tomográfica abdominal, dosagem de CEA e estudo radiográfico de tórax. A comprovação da inexistência de envolvimento além da submucosa indicava a TEM. Além disso, a avaliação histológica pré-operatória incluiu: 1) definição da natureza da lesão neoplásica conforme os critérios da OMS e UICC-TNM ${ }^{(30)}$; 2) critérios histomorfológicos de estimativa de risco de metástases angiolinfáticas associada à profundidade da invasão do tumor primário (uT), ao grau de diferenciação celular histológica conforme a OMS, à presença ou ausência de linfangiose carcinomatosa (baseado na constatação de células tumorais no interior de cavidades revestidas por endotélio e sem elementos musculares na parede $\left.{ }^{(30)}\right)$.

O estádio uTNM era feito no pré-operatório baseado nos critérios ultra-sonográficos de HILDEBRANDT et al. ${ }^{(11)}$, sendo a) uTo, lesão restrita à mucosa; b) uT1, lesão restrita à submucosa; c) uT2, lesão restrita à muscular própria; d) uT3, lesão restrita à gordura perirretal; e) uT4, lesão invadindo órgãos adjacentes; e) No, sem invasão linfonodal; f) N1, com invasão linfonodal.

\section{O procedimento operatório (TEM)}

O preparo do colo era feito com lavagem anterógrada de 2000 mL de manitol a 10\%, 6 a 8 horas antes da operação. O procedimento anestésico era realizado com anestesia peridural ou geral, na dependência da gravidade clínica e da melhor indicação para o doente, pelo Serviço de Anestesiologia. Antibioticoprofilaxia com cefoxitina na dose de $2 \mathrm{~g}$ intravenosa era administrada na indução anestésica.

O doente era posicionado na mesa operatória de modo variado, na dependência da localização do tumor que deveria estar, sempre que possível, com a maior área localizada posteriormente, às 6 horas. Esta posição era definida no pré-operatório.

O procedimento cirúrgico começava pela localização exata do tumor. Através do retoscópio de Buess (Richard Wolf Co., Knittlingen, Germany), com $40 \mathrm{~mm}$ de diâmetro e comprimento variável entre 12 e $20 \mathrm{~cm}$, introduzido no reto com um mandril próprio do equipamento, o tumor era visualizado, inicialmente através de um diafragma de vidro. Para que se pudesse ter plena observação, realizava-se insuflação manual para distensão do reto.

Após ser obtida a visualização adequada do tumor, o retoscópio era fixado em suporte apropriado (Martin). O diafragma de vidro, 
que serviu para acesso à visualização, era substituído pelo portal de trabalho. Este portal era composto de: a) eletrocautério de alta freqüência que regulava automaticamente o arco de voltagem e a intensidade elétrica, para proporcionar controle eletrônico instantâneo de corte (Erboton ICC 350M; Erbe Elektromedizin, Alemanha); b) telescópio binocular rígido de visão tridimensional estereoscópica; c) uma ótica de documentação acoplada ao equipamento para que a equipe cirúrgica acompanhasse o procedimento. A imagem produzida é bidimensional, como a da videolaparoscopia, diferente daquela do cirurgião da TEM, que é tridimensional (Figura 2).

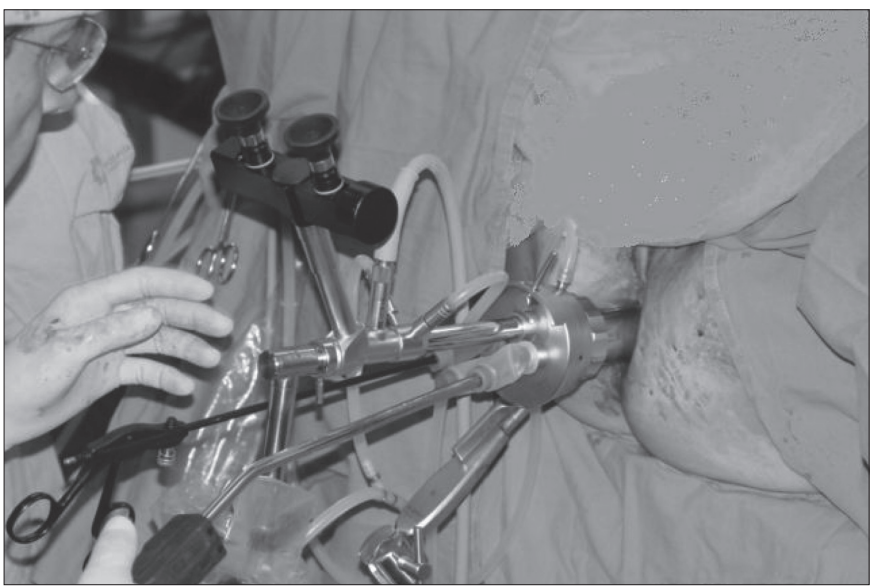

FIGURA 2. Retoscópio de Buess montado para a realização do ato operatório (Richard Wolf Company, Knittlingen, Alemanha)

O campo operatório, a seguir, era ampliado pela insuflação de dióxido de carbono, por acesso a ele destinado no equipamento, mantido eletronicamente em pressão constante e uniforme de $15 \mathrm{~mm} \mathrm{Hg}$ para permitir bom controle visual do campo cirúrgico e segurança quanto aos limites de ressecção. Sistemas de irrigação e aspiração eram, então, adicionados ao portal e interligados a insuflador por uma bomba de circulação. $\mathrm{O}$ bisturi eletrônico era próprio do sistema TEM e usado no procedimento com múltiplas funções, que eram comandadas por uma bomba pneumática. Esta era controlada por pedal que ordenava o corte, a coagulação, a aspiração e a irrigação, de acordo com a necessidade do cirurgião.

A incisão cirúrgica, diferentemente das ressecções endoscópicas, era feita interessando às camadas mucosa, submucosa, muscular e gordura perirretal (ressecção total da parede), com margem cirúrgica radial mínima de $5 \mathrm{~mm}$ para os adenomas e $10 \mathrm{~mm}$ para os carcinomas. A área cruenta remanescente era fechada com fio monofilamentado 2-0, agulha de $2 \mathrm{~cm}$ e clipes de prata.

Revisão minuciosa do sangramento e perfeito fechamento da área ressecada, finalizava o procedimento.

\section{Preparacão dos espécimes e estudo anatomopatológico}

O tecido excisado era imediatamente fotografado, distendido em uma superfície lisa e fixado em solução de formalina a $10 \%$.

Após $48 \mathrm{~h}$ de fixação, o espécime era refotografado, pintado com tinta nankin com duas cores que permitiam a identificacão, no exame histológico, das margens ou limites de ressecção radial e perimetral, sendo realizado desenho esquemático com registro detalhado das amostras colhidas, que eram numeradas e, a seguir, o espécime era totalmente incluído (Figuras 3 e 4). Para garantir

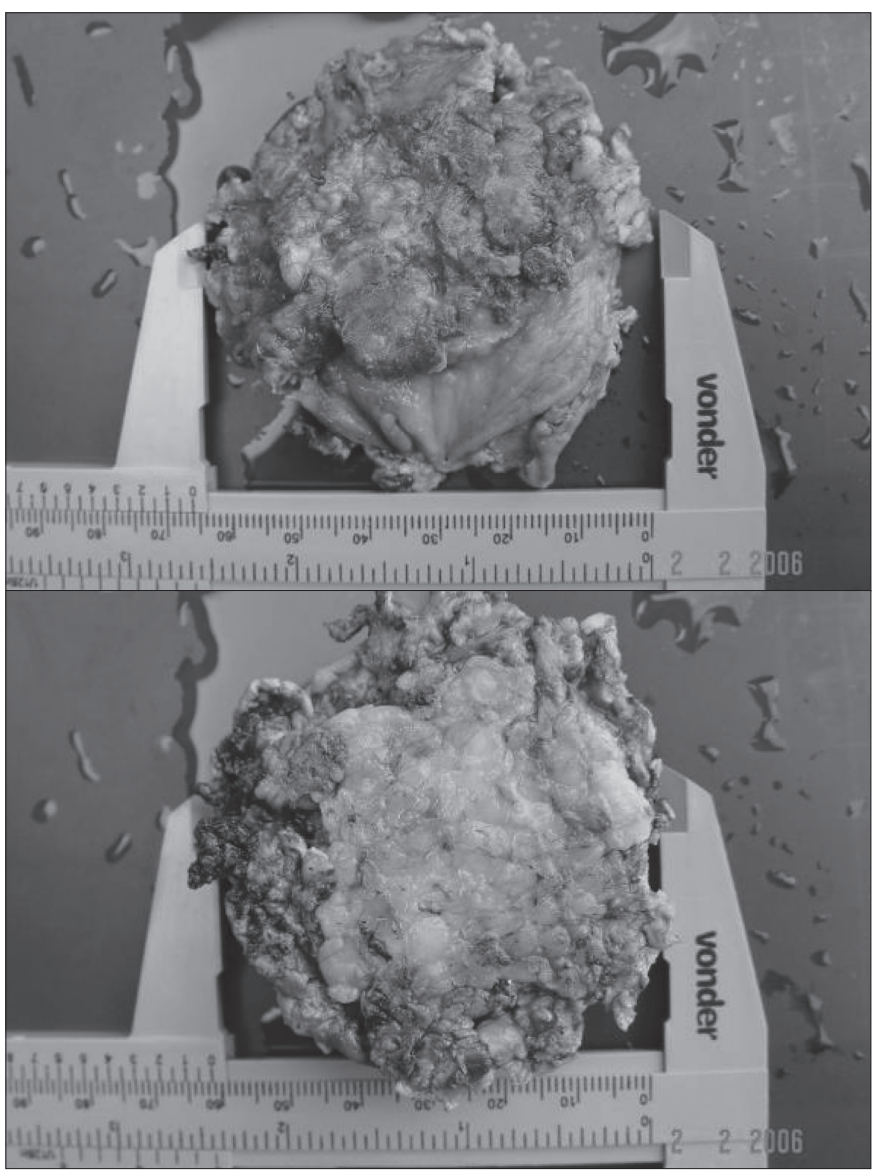

FIGURA 3. Aspecto macroscópico da ressecção mostrando margem oncológica circunferencial maior do que $1 \mathrm{~cm}$

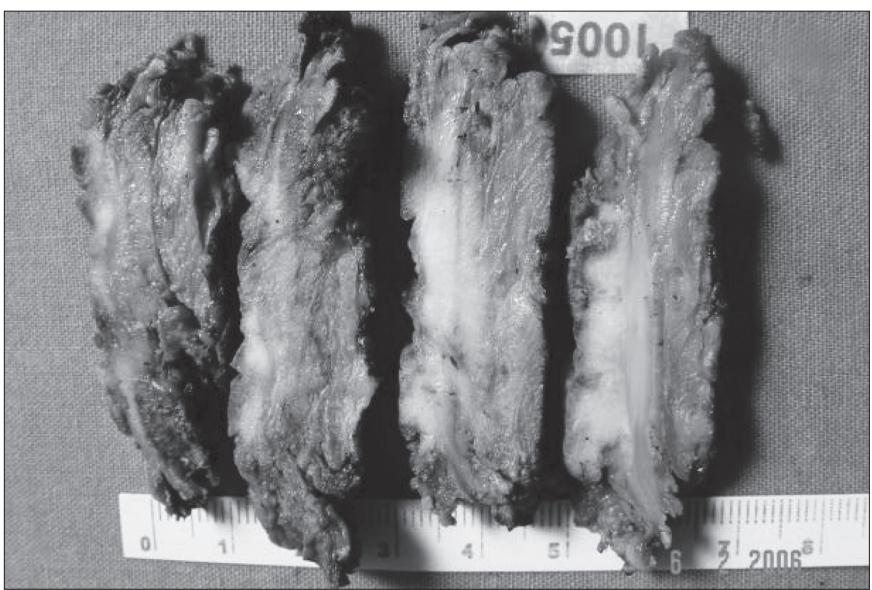

FIGURA 4. Aspecto macroscópico dos cortes corados com tinta nanquim em 2 cores para o estudo histológico - ressecção total da parede do reto 
informações relevantes ao patologista, utilizava-se formulário padrão que mencionava sistematicamente a localização, o tipo macroscópico (plano, pediculado ou ulcerado), o número de lesões excisadas e a impressão do uTNM $^{(11)}$.

Os cortes histológicos foram corados com hematoxilina-eosina (H-E) (Figura 5). Para se estimar o risco de metástase linfogênica foi usado o termo de baixo risco, para os adenocarcinomas de graus G1 e G2 sem linfangiose carcinomatosa. Os tumores G3 e G4 e aqueles com evidência de linfangiose carcinomatosa eram classificados como de alto risco (coloracão em H-E). Naqueles onde havia vários graus de diferenciação associados, o grau menos diferenciado era utilizado. Para melhor se visualizar blocos epiteliais diminutos e infiltrativos e para confirmar o fenômeno de invasão vascular linfática, foi realizado estudo imunoistoquímico com anticorpos tipo CD34 e citoqueratinas. O laudo anatomopatológico dos espécimes da TEM continham: a) quantidade de camadas envolvidas; b) natureza da lesão; c) profundidade da invasão; d) tipo histológico e graduação; e) avaliação de invasão vascular; f) avaliação da margem radial e perimetral. Esses critérios foram baseados na proposta de $\operatorname{HERMANECK}^{(8,9,10)}$. O registro eventual da categoria tumor residual foi feito nos casos tratados por TEM que apresentaram recidivas e foram documentados no protocolo dos submetidos a re-operacão da seguinte forma: residual ausente $=\mathrm{R} 0$, residual microscópico $=\mathrm{R} 1$; residual macroscópico $=\mathrm{R} 2$. A classificação $\mathrm{R}$ refere-se ao tumor primário, linfonodos regionais e metástases à distância ${ }^{(30)}$.

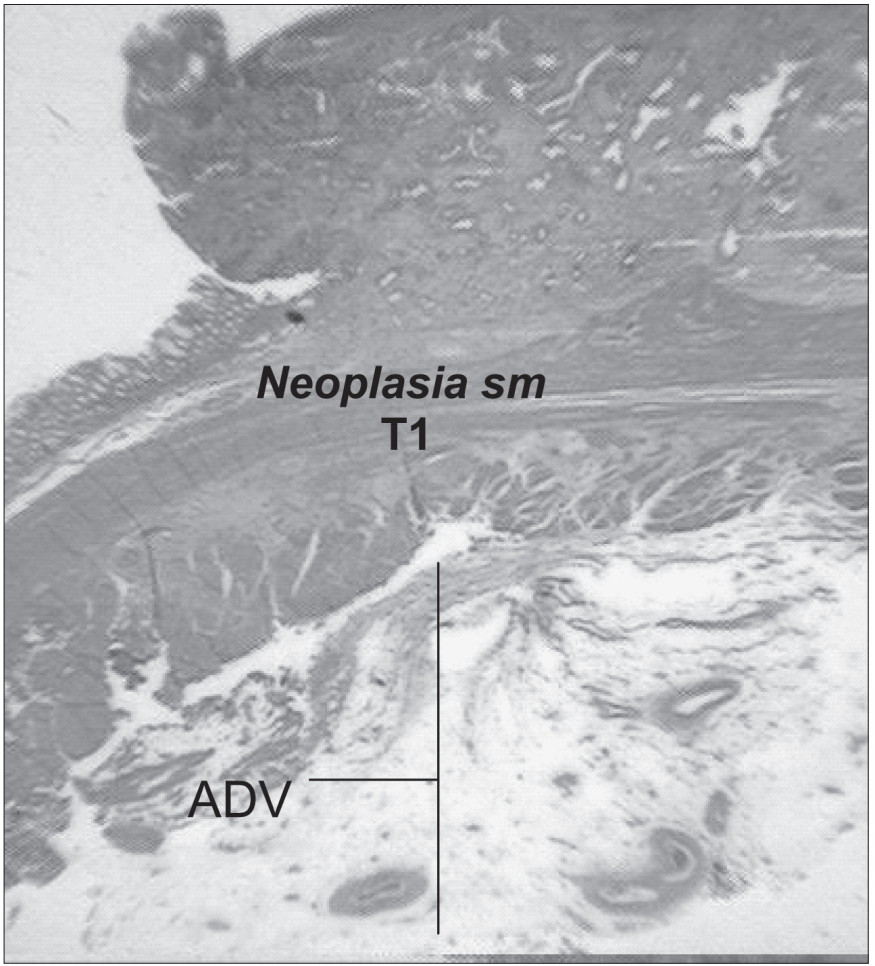

FIGURA 5. Aspecto histológico com coloração H-E mostrando o tumor invadindo a submucosa. Ressecção total da parede do reto

\section{Cuidados pós-operatórios e de seguimento}

Retoscopia flexível era feita no $5^{\circ}$ e no $21^{\circ}$ dia pós-operatório para se verificar a permeabilidade da anastomose.

O seguimento endoscópico pós-operatório era feito nos adenomas, no $3^{\circ}$ mês e de 6 em 6 meses, nos primeiros 2 anos. Se não houvesse a presença de novos pólipos, era preconizado de 5 em 5 anos. Nos carcinomas, de 3 em 3 meses nos 2 primeiros anos, e de 6 em 6 meses nos 3 anos subseqüentes e consistia de múltiplas biopsias na área anastomótica. Passado o período de risco de recidiva, adotou-se o rastreamento feito para a população em geral, isto é, de 5 em 5 anos. O controle do CEA era feito de 6 em 6 meses durante 5 anos, somente nos carcinomas.

\section{RESULTADOS}

A maior incidência dos tumores foi observada entre 60 e 79 anos (28 pacientes).

A neoplasia intra-epitelial de alto grau (carcinoma in situ, carcinoma intramucoso e carcinoma superficial) foi mais prevalente, ocorrendo em 26 pacientes, seguida pelos carcinomas pT1 em 9 , adenomas em 6, carcinoma pT2 em 4 e carcinóide em 2. Observou-se uma recidiva (1/50) 18 meses após a TEM e dois tumores residuais diagnosticados no $3^{\circ}$ mês do pós-operatório.

Os tumores foram mais prevalentes na parede posterior do reto $(n=34)$, o que determinou igualmente predominância da posição de litotomia no ato operatório. As outras posições utilizadas foram o decúbito lateral esquerdo em 9 vezes, lateral direito em 6, e ventral em 5 .

As complicações observadas foram divididas em maiores e menores (Tabela 1). A mais grave foi uma fístula retovaginal em uma re-resseccão de um carcinoma pT1. Sangramento pós-operatório ocorreu em dois pacientes, um deles no pósoperatório imediato e o outro no $8^{\circ}$ dia. Nenhum deles necessitou reintervencão. A deiscência parcial da anastomose foi observada em três, todos elas de lesões extensas e com anastomose retoanal. $\mathrm{O}$ enfisema de escroto foi observado em um paciente, em que foi realizada ressecção total da parede anterior do reto. Incontinência temporária a gases foi relatada em três pacientes com anastomoses retoanais e que se resolveram espontaneamente. Tromboflebite

TABELA 1. Microcirurgia endoscópica transanal em 28 pacientes (abril de 2002 a abril de 2006)

\begin{tabular}{lc}
\hline Complicações & Tratamento \\
\hline Fístula retovaginal $(\mathrm{n}=1)($ maior $)$ & Conservador \\
Sangramento pós-operatório $(\mathrm{n}=2)$ & Conservador \\
Deiscência de anastomose $(\mathrm{n}=3)$ & Conservador \\
Enfisema de escroto $(\mathrm{n}=1)$ & Conservador \\
Incontinência fecal transitória $(\mathrm{n}=3)$ (neuropraxia) & Conservador \\
Tromboflebite superficial $(\mathrm{n}=1)$ & Conservador \\
Dificuldade urinária $(\mathrm{n}=2)$ & Cateter urinário (1) \\
Abertura do peritônio $(\mathrm{n}=1)$ & Sutura transanal \\
\hline
\end{tabular}


superficial ocorreu em um paciente com predisposição, apesar dos cuidados de tromboprofilaxia. Disúria a dificuldade urinária foi observada em dois pacientes no pós-operatório imediato. Uma abertura inadvertida da cavidade foi tratada por via transanal. Um paciente de 92 anos com adenocarcinoma $\mathrm{pT} 2 \mathrm{~N} 1$ foi a óbito no $3^{\circ}$ dia pós-operatório (dia da alta) por embolia pulmonar maciça (Tabela 1).

\section{DISCUSSÃO}

A TEM é um método especial utilizado no tratamento local dos tumores do reto, o qual exige capacitação em centros de treinamento e deve ser praticado em hospitais de referência, pois o número de doentes a ser operado não é grande, o que torna a curva de aprendizado lenta e difícil. Envolve uma série de cuidados abrangentes que vão além do ato operatório, incluindo na sua realização, um grupo de especialidades médicas diferentes e que devem cumprir criteriosamente protocolos pré-estabelecidos para esse fim. Deve ser considerada como procedimento cirúrgico endoscópico, realizado através de um conjunto de equipamentos sofisticados que não devem ser confundidos com insufladores e instrumentais convencionais da videolaparoscopia. Permite ao cirurgião visão binocular, portanto tridimensional estereoscópica ampliada. Seu equipamento inclui sistema eletrônico de insuflação uniforme que dá controle visual excelente do campo operatório durante todo o procedimento. O instrumental é especialmente desenhado para ela, baseado no paralelismo dos movimentos, para que se obtenha o maior desempenho quando se trabalha em um tubo de $40 \mathrm{~mm}$. Tudo isso permite segurança quanto aos limites de ressecção, o que se traduz por baixas taxas de recidiva, quando comparado aos métodos convencionais de tratamento local ${ }^{(1,3)}$.

Finalmente a TEM proporciona a ressecção em bloco de toda a parede retal e gordura perirretal, o que a torna procedimento oncológico, especialmente naquelas lesões sésseis maiores do que $4 \mathrm{~cm}$, onde a presença de carcinoma incidental pT1 está em torno de $20 \%{ }^{(17,24)}$. O presente estudo foi prospectivo, nãorandômico e não poderia sê-lo diferente, devido aos critérios rígidos de inclusão e exclusão. Não seria ético submeter pacientes passíveis de ressecção local curativa com preservação do reto a um procedimento mutilador de controle, uma vez que os dois procedimentos poderiam proporcionar a mesma chance de cura.

As complicações foram baixas em cifras percentuais. Foi observada uma fístula retovaginal na re-ressecção de um carcinoma pT1, realizada previamente com alça de polipectomia, cujas margens ficaram coincidentes com a neoplasia. A cauterização excessiva do leito cruento deve ter sido a causa do seu aparecimento. Foi instituído tratamento conservador, com conseqüente fechamento da fístula ${ }^{(20)}$.

Outra paciente de 37 anos teve como diagnóstico carcinoma pT2 N0 de baixo risco, tendo se recusado ao tratamento radical convencional e à radioterapia e à quimioterapia. Encontra-se atualmente em seguimento de 24 meses, sem recidiva. Embora a literatura mostre nesses casos recidiva de até $22 . \%$ sem tratamento complementar, já existem relatos onde casos escolhidos de carcinoma pT2 foram submetidos a tratamento local por TEM, com bons resultados. São lesões bem diferenciadas menores do que $4 \mathrm{~cm}$, sem invasão angiolinfática que respondem bem à radioterapia pré-operatória. Já foi observado que, em seguimento tardio de 10 anos, não houve diferença significativa na sobrevida, quando comparados os pacientes submetidos a TEM aos com procedimento cirúrgico radical convencional ${ }^{(16)}$. Naquele caso a ultra-sonografia pré-operatória foi de pT1N0, o que demonstra que, às vezes, torna-se difícil determinar com exatidão a profundidade da invasão.

O diagnóstico definitivo muitas vezes só é feito no pósoperatório com a retirada em bloco da peça. A literatura mostra que a ecografia endorretal tem precisão próxima de $90 \%$, com margem de erro de $10 \%$ para mais ou para menos. Poderão ocorrer problemas com a ecografia endorretal quando o tumor estiver próximo do canal anal e quando existirem úlceras conseqüentes de tratamentos anteriores. Particularmente, cuidado especial deve-se ter quando os tumores se encontrarem acima da reflexão peritonial e não puderem ser alcançados com o transdutor rígido. O que não se pode admitir atualmente é que o tratamento dos tumores do reto, ressecção local ou a operação radical convencional, seja feito sem estádio prévio adequado ${ }^{(11)}$.

Foram observadas três deiscências de anastomose, sem significância clínica em lesões extensas próximas à linha pectínea.

Quanto maior a área cruenta e maior a proximidade da linha pectínea, maior a probabilidade de deiscência, sendo ela mais freqüente entre 3 e $5 \mathrm{~cm}$ da margem anal e menos freqüente entre 9 e $11 \mathrm{~cm}^{(20)}$. Sangramento pós-operatório foi observado em dois doentes que não necessitaram reintervenção.

A incontinência temporária foi observada em seis pacientes. $\mathrm{Na}$ realidade o que se observa clinicamente é uma urgência defecatória, especialmente nas lesões muito próximas da linha pectínea. $\mathrm{O}$ que parece é que esses pacientes fazem neuropraxia do esfíncter interno, reduzindo temporariamente a sua função. Esta lesão pode ser determinada pela insuflação prolongada e dilatação forçada na introdução do retoscópio. A literatura mostra que não há prejuízo definitivo da função esfincteriana e que os estudos manométricos pré e pós-operatórios comparados não apresentaram diferenças significativas após 90 dias $^{(4,15)}$. Uma abertura inadvertida da cavidade foi tratada por sutura via transanal.

Realizou-se ressecção circunferencial do reto com anastomose retoanal em um doente. Os tumores carcinóides foram tratados pela TEM, com a ressecção total da parede do reto. Um deles tinha sido ressecado previamente por polipectomia endoscópica. Era lesão séssil e as margens de ressecção ficaram coincidentes com a lesão. Existe tendência atual em se dizer que a ressecção desses tumores pode ser feita pela polipectomia convencional, afirmação que não deve ser aceita porque o carcinóide já nasce pT1 na submucosa e requer ressecção transmural. Portanto, a ressecção endoscópica com alça só se aplica às lesões pediculadas ${ }^{(12,24)}$.

Dois pacientes apresentaram tumor residual 3 meses após a TEM, sendo um deles submetido ao tratamento complementar com ablação por plasma de argônio e o outro por polipectomia em alça. DEMARTINES et al. ${ }^{(5)}$, em trabalho de revisão sobre a recurrência dos adenomas após TEM, verificaram que a média é 
de 5,8\% e a morbidade em torno de $10 \%$, inferior ao da ressecção anterior. Paciente de 92 anos com tumor pT2 N1, ASA 4 foi submetido a ressecção paliativa. A operação foi minimamente invasiva, possibilitando inclusive a avaliação do linfonodo identificado na ultra-sonografia prévia, que confirmou estar comprometido no exame histopatológico ${ }^{(24)}$. Um tumor pT1 de baixo risco apresentou recidiva 18 meses após a ressecção local, sendo submetido a ressecção anterior curativa com diagnóstico final de pT3NO. Neste caso houve suspeita de invasão angiolinfática na $\mathrm{H}-\mathrm{E}$ que foi subestimada na primeira operação. Como um novo recorte para estudo imunoistoquímico não foi conclusivo, evitou-se tratamento adjuvante. Encontra-se em seguimento, após a segunda operação, há 18 meses sem recidiva.

$\mathrm{O}$ risco de recidiva no carcinoma $\mathrm{pT} 1$ de baixo risco, tratado pela TEM, deve ser analisado, comparando-o ao da operação radical convencional curativa, especialmente aquela realizada com dissecção romba, e com a morbimortalidade dos procedimentos. De acordo com estudos publicados na literatura, a média da mortalidade na ressecção anterior está em torno de 5\% aos 50 anos, e aumenta significativamente com a idade, chegando a 13\% naqueles acima de $80^{(13)}$. Nestes, freqüentemente existem fatores de risco adicionais. Considerando-se que tumores nos estádios iniciais têm período longo sem recurrência, pode-se admitir que muitos dos idosos não viverão a tempo de experimentar recidiva. Assim, são candidatos ótimos para o tratamento local ${ }^{(31)}$.

Quando se estende esses conceitos aos pacientes jovens com pT1 de baixo risco, estudos com seguimento longo sugerem recidiva em torno de $5 \%{ }^{(20)}$. Dessa forma, para $95 \%$ dos pacientes nesta condição, a operação radical convencional é desnecessária. Entretanto, se a recidiva ocorrer, embora esses doentes sejam operados num estádio mais avançado, ainda haverá chance de cura maior do que $50 \%$ em segunda operação ${ }^{(6)}$, o que ocorreu com um dos pacientes desta casuística.

Em estudo prospectivo randômico realizado por WINDE et al. ${ }^{(32)}$, em 1996, onde foram comparados os resultados tardios da ressecção local por TEM versus ressecção anterior em carcinomas pT1, observaram que a perda sangüínea operatória, a mortalidade, o tempo de hospitalização e o uso de analgésicos e opiáceos foram menores na TEM do que na ressecção anterior. Quando compararam a curva de sobrevida nos dois grupos não houve diferença significativa. POLGLASE ${ }^{(27)}$, em estudo onde foi analisada a recidiva do tumor do reto após retossigmoidectomia, especialmente com dissecção romba, verificou que ela varia entre 3.1 e 5.3, resultado semelhante ao obtido com a TEM nos estádios iniciais, mas com menor morbimortalidade. Por outro lado, quando se compara a recidiva da TEM com os $23 \%$ proporcionados pela ressecção local convencional, deve-se considerar proibitivo este método ${ }^{(26)}$.

Com taxa de recidiva média de $10 \%$, o prognóstico dos pacientes com carcinoma $\mathrm{pT} 1$ de baixo risco operados por TEM e que se submeteram a segundo tratamento cirúrgico convencional, parece ser sempre pior $^{(1)}$. Entretanto, isso se deve a dois fatores: as células tumorais se espalharam ou ficou lesão residual na primeira operação, o que só pode ser confirmado através de um estudo randômico prospectivo.

Em 1994, SALM et al. ${ }^{(29)}$ relataram experiência com a TEM na Alemanha. Foram realizadas 1.900 operações, sendo 1.411 adenomas e 433 carcinomas. Dessas operações 286 foram com intenção curativa e 147 com intenção paliativa. A taxa de conversão da TEM para laparotomia variou com a experiência do cirurgião $0^{(2,6,27,30)}$. A ressecção radical do reto como segunda opção foi realizada em $5,7 \%$, nos casos de carcinoma avançado ao exame histológico. As complicações ocorreram em 120 pacientes $(6,3 \%), 77(4,0 \%)$ tratados conservadoramente e 43 (2,3\%), com procedimento cirúrgico. A mortalidade foi de $0,2 \%$.

Dois pacientes com adenomas maiores do que $4 \mathrm{~cm}$, apresentaram-se, ao diagnóstico final, com focos isolados de carcinoma $\mathrm{pT} 1$. A literatura mostra a presença incidental desse carcinoma em 20\% dessas lesões. Por isso, recomenda-se que eles sejam sempre tratados com ressecção transmural ${ }^{(20)}$. MORSCHEL et al. ${ }^{(25)}$, comparando o diagnóstico pré e pósoperatório das lesões ressecadas, chamaram atenção sobre a presença de adenocarcinoma, em $23 \%$ dos pólipos menores do que $3 \mathrm{~cm}$.

A média de complicações após TEM que requer intervenção cirúrgica $(2,3 \%)$ é muito inferior àquela apresentada pela operação radical convencional ${ }^{(29)}$.

A pergunta a ser respondida é: poderia a TEM ser utilizada com sucesso em carcinomas com estádio mais avançado? A literatura já mostra resultados animadores com sua aplicação nos carcinomas pT2NO submetidos a radioterapia e quimioterapia pré-operatórias $^{(3,16,20,28,29)}$.

\section{CONCLUSÃO}

As indicações da TEM no tratamento dos adenomas sésseis, das neoplasias intra-epiteliais de alto grau e do carcinoma pT1 de baixo risco do reto, são precisas, além de apresentar benefícios com resultados oncológicos comparáveis aos das operações radicais convencionais. 
Moraes RS, Malafaia O, Telles JEQ, Trippia MA, Buess GF, Coelho JCU. Transanal endoscopic microsurgery in the treatment of rectal tumors: a prospective study in 50 patients. Arq Gastroenterol. 2008;45(4):268-74.

ABSTRACT - Background - The medical literature accepts local resection as a valuable option in selected cases of rectal tumors. Selection of patients requires an exact perioperative estimation of risks with clinical and histopathological examination. Transanal endoscopic microsurgery aims a safe resection of the tumoral area which leads up to the cure. Aim - To evaluate transanal endoscopic microsurgery results in a follow-up time of 18 months. Methods - From April 2002 to April 2006, 50 patients with rectal tumors were submitted to transanal endoscopic microsurgery, chosen by clinical history and lesion characteristics. The inclusion criteria were: sessile adenomas larger than $3 \mathrm{~cm}$ and smaller than $8 \mathrm{~cm}$, not circumferentially distributed; intra epithelial neoplasia of high degree; and rectal carcinoma pT1, and special cases of pT2. All these rectal tumors were submitted to the same surgical act. Results - The final histopathological results reveal 9 adenoma, 26 intra-epithelial neoplasia of high degree, 13 carcinoma (9 pT1-4 pT2) and 2 carcinoid. The lowest age was 25 and the higher, 92 years-old. The smallest resected tumor had $64 \mathrm{~mm}^{2}$ (carcinoid) and the largest (adenoma), $90 \mathrm{~cm}^{2}$. Operating time was in average $90 \mathrm{minutes}$ and the overall time statement, 5 days. There was one death not related with the method. One patient with low risk carcinoma pT 1 presented recurrence 18 months after transanal endoscopic microsurgery and was submitted to curative rectosigmoidectomy. It was proven a residual tumor after local surgery in two patients and the most important complication was one recto-vaginal fistula. The overall complications rate was $9 \%$. Conclusion - Today transanal endoscopic microsurgery is chosen as the ideal technique for the treatment of sessile adenomas, intraepithelial neoplasia of high degree and rectal carcinoma pT1.

HEADINGS - Rectal neoplasms, surgery. Microsurgery. Proctoscopy.

\section{REFERÊNCIAS}

1. Azimuddin K, Riether RD, Stasik JJ, Rosen L, Khubchandani IT, Reed JF 3rd. Transanal Endoscopic Microsurgery for Excision of Rectal lesions: technique and initial results. Surg Laparosc Endosc Percutan Tech 2000; 10: 372-8.

2. Buess G, Kipfmuller K, Hack D, Grussner R, Heintz A, Junginger T. Technique of transanal endoscopic microsurgery. Surg Endosc. 1988;2:71-5.

3. Buess G, Kipfmuller K, Ibald R, Heintz A, Hack D, Braunstein S, Gabbert H, Junginger T. Clinical results of transanal endoscopic microsurgery. Surg Endosc. 1988;2: 245-50.

4. Cataldo P, O’Brien S, Osler T. Transanal endoscopic microsurgery: a prospective evaluation of funcional results. Dis Colon Rectum. 2005:48:1366-71.

5. Demartines N, von Flüe MO, Harder FH. Transanal endoscopic microsurgical excision of rectal tumors: indications and results. World J Surg. 2001:25:870-5.

6. Graham RA, Garnsey L, Jessup JM. Local excision of rectal carcinoma. Am J Surg. 1990;160:306-12.

7. Haring R, Karavias T, Konradt J. Posterior proctorectotomy. Chirurg. 1978;49: 265-71.

8. Hermanek P. Polypectomy in the colorectum histological and oncological aspects. Endoscopy. 1983;15(Suppl 1):158-61.

9. Hermanek P. Colorectal carcinoma: histopathological diagnosis and staging. Baillieres Clin Gastrenterol. 1989;3:511-29.

10. Hermanek P. Malignant polyps - pathological factors governing clinical management. Curr Top Pathol. 1990;81:277-93.

11. Hildebrandt U, Feifel G, Zinmermann FA, Goebbels R. Significant improvement in clinical staging of rectal carcinoma with a new intrarectal ultrasound scanner. J Exp Clin Cancer Res. 1983;2:53-9.

12. Ishikawa K, Arita T, Shimoda K, Hagino Y, Shiraishi N, Kitano S. Usefullness of transanal endoscopic surgery for carcinoid tumor in the upper and middle rectum. Surg Endosc. 2005;19:1151-4.

13. Kessler H, Hermanek P Jr, Wiebelt H. Operative mortality in carcinoma of rectum. Results of the German Multicentre Study. Int J Colorectal Dis. 1993;8:158-66.

14. Kraske P. Zur extirpation hochsitzender Mastdarm krebsen. Verh Dtsh Ges Chir. 1885;14:464-74.

15. Kreis M, Jehle EK, Haug V, Manncke K, Buess FG, Becker H, Starlinger MJ. Funcional results after transanal endoscopic microsurgery. Dis Colon Rectum. 1996;39: 1116-21.

16. Lezoche E, Guerrieri M, Paganini AM, Feliciotti F. Long-term results of pacients with pT2 rectal cancer treated with radiotherapy and transanal endoscopic microsurgical excision. World J Surg. 2002;26:1170-4.

17. Maslekar S, Beral DL, White TJ, Pillinger SH, Monson JR. Transanal endoscopic microsurgery: where are we now?. Dig Surg. 2006;23:12-22.
18. Mason AY. Surgical access to the rectum - a transsphincteric exposure. Proc R Soc Med. 1970;63:91-4

19. Mellgren A, Sirivongs P, Rothenberger DA, Madoff RD, Garcia-Aguilar Jr. Is local excision adequated therapy for early rectal cancer? Dis Colon Rectum. 2000;43: 1064-71.

20. Mentges B, Buess G, Raestrup H, Manncke K, Becker HD. TEM results of tuebingen group. End Surg Allied Technol. 1994;2:247-50.

21. Moraes RS, Buess G, Campos ACL, Malafaia O, Marinho Jr CH, Coelho JCU. Transanal endoscopic microsurgery in the treatment of superficial rectal cancer. Report of a case. ABCD Arq Bras Cir Dig. 2002;15:36-9.

22. Moraes RS, Campos ACL, Telles JEQ, Coelho JCU. Microcirurgia endoscópica transanal (TEM) no contexto da cirurgia minimamente invasiva para o tratamento dos tumores do reto. ABCD Arq Bras Cir Dig. 2003:16:3-5.

23. Moraes RS, Telles JEQ, Trippia M, Bizinelli SL, Telles GHQ. Estudo prospectivo do tratamento dos tumores retais por microcirurgia endoscópica transanal - Experiência multidisciplinar. Rev Col Bras Cir. 2003;30:28.

24. Moraes RS, Ferrarini DD, Telles JEQ, Scaccia E, Moura G. Ressecção oncológica minimamente invasiva de tumor carcinóide do reto por microcirurgia endoscópica transanal (TEM) [resumo]. In: GASTREN 2004. Recife: Anais da VI Semana do Aparelho Digestivo 2004. p.80.

25. Morschel M, Heintz A, Junginger TM. Risk of malignant degeneration of preoperatively classified benign large sessile retal polyps. A comparison with adenoma size. Zentralbl Chir. 1999;124:226-9.

26. Muto T, Bussey HJ, Morson BC. The evolution of cancer of colon and rectum. Cancer 1975;36:2251-70

27. Polglase AL, McMurrick PJ, Tremayne AB, Bathal PS. Local recurrence after curative anterior resection with principally blunt dissection for carcinoma of rectum and rectosigmoid. Dis Colon Rectum. 2001:44;947-53.

28. Saclarides TJ. What's new in ACS surgery: principles and practice. Transanal Endoscopic Microsurgery. Bull Am Col Surg. 2004. 89:36-7.

29. Salm R, Lampe H, Bustos A, Matern U. Experience with TEM in Germany. Endosc Surg Alied Technol. 1994;2:251-4.

30. UICC International Union Against Cancer. TNM Supplement 1993: A commentary on uniform use. In: Hermanek P, Henson DE, Hutter RVP, Sobin LH, editors. Berlin: Springer; 1993.

31. Welch JP, Donaldson GA. Detection and treatment of recurrent cancer of the colon and rectum. Am J Surg. 1978;135:505-11.

32. Winde G, Nottberg H, Keller R, Schmid KW, Bünte H. Surgical cure for early rectal carcinomas (T1). Transanal endoscopic microsurgery vs. anterior resection. Dis Colon Rectum. 1996:39:969-76.

Recebido em 2/8/2007. Aprovado em 25/4/2008 